\title{
21. THE SEPARATION OF SMALL PARTICLES FROM METEOR BODIES, AND ITS INFLUENCE ON SOME PARAMETERS OF METEORS
}

\author{
A. N. SimonenKo \\ (O.J. Smidt Institute of Geophysics, Academy of Sciences of the U.S.S.R., Moscow)
}

The separation of very small particles from meteor bodies as a part of the process of their ablation in the Earth's atmosphere is assumed by many investigators. But when the particle is small relative to the meteor body the path of the particle is a priori thought to be small relative to the length of the meteor path, i.e. the particle is expected to evaporate in close proximity to the point of separation. This assumption is not acceptable after McCrosky's work (1958) that has shown for one particular case the small particles $\left(m=10^{-5}-10^{-6} \mathrm{~g}\right)$ go a long path before their mass is exhausted. The assumption made until now has caused incorrect conclusions, e.g. the luminosity at any point of the meteor path is proportional to the mass ablated from the meteor body at that point (Kramer, 1965).

On the other hand, the behaviour of small particles separated from the meteor body after the beginning of its intensive evaporation is believed to be similar to that of micrometeorites, i.e. small particles that individually penetrate into the atmosphere (Kramer, 1965). The latter were investigated by Whipple $(1950,1951)$. He pointed out that the intensive evaporation of micrometeorites, occurs, if at all, after their initial pre-atmospheric velocity has decreased considerably. Micrometeorites keep the major part of their initial mass and fall to Earth as cosmic dust. The bigger meteor bodies are retarded very little at great heights. Down to the height of the beginning of intensive evaporation, their velocity is practically unchanged. If a small particle is separated from the meteor body after the beginning of intensive evaporation, it is (i) strongly heated (separates from a heated surface) and (ii) goes into the relatively dense layers of the atmosphere with a velocity equal to that of the meteor body, i.e. approximately the initial velocity of the meteor. The energy flux received by the particle from the air is much greater than the energy loss of the particle by thermal radiation, even assuming the shielding of the particle by its vapor. The intensive evaporation of the particle begins immediately after its separation from the meteor body and proceeds until all particle mass is exhausted.

In the case of the absence of fragmentation of small particles during their flight*,

* The fragmentation of small particles is not possible, on physical grounds. 
to study their behaviour we can start from the basic equations of meteor theory for the case of a solid unfragmented body, in particular from the drag equation and the luminosity equation. For a spherical particle

$$
\begin{aligned}
\frac{\mathrm{d} V}{\mathrm{~d} t} & =-\Gamma A \delta^{-2 / 3} \rho m^{-1 / 3} V^{2}, \\
I & =\frac{2 \tau_{0}}{9 \pi} \Gamma A \sigma \frac{m_{0}^{2 / 3}}{\delta^{2 / 3}} \rho V^{6} \exp \left[-{ }_{3}^{\sigma}\left(V_{0}^{2}-V^{2}\right)\right],
\end{aligned}
$$

and the set of equations giving the change of velocity versus the length of the path is:

$$
\begin{gathered}
l=\mathbf{H} \sec z \ln \rho_{2} / \rho_{1}, \\
\rho_{2}-\rho_{1}=\frac{m^{1 / 3} \delta^{2 / 3} \cos z}{2 \Gamma A \mathbf{H}} \exp \left(\begin{array}{c}
\sigma V_{0}^{2} \\
6
\end{array}\right)\left[E i\left(\begin{array}{c}
\sigma V_{0}^{2} \\
6
\end{array}\right)-E i\left(\begin{array}{c}
\sigma V^{2} \\
6
\end{array}\right)\right] .
\end{gathered}
$$

The behaviour of a particle separated from the meteor body is determined mainly by the initial size of the particle and the height of separation, and to a lesser extent by its initial velocity and direction of incidence (Simonenko, 1968).

We carry out the computations of the length of the path, $L$, of the particles with different heights of separation, $H_{1}$, and different initial velocity, $V_{0}$. The parameters of the atmosphere are assumed to be $\rho_{0}=5 \times 10^{-3} \mathrm{~g} / \mathrm{cm}^{3}, \mathbf{H}=6.5 \mathrm{~km}$. The particles are assumed spherical with initial radius $r_{0}=0.01 \mathrm{~cm}$, density $\delta=3.5 \mathrm{~g} / \mathrm{cm}^{3}$, and a trajectory at vertical incidence. These particles are called 'standard' ones. The velocity at which the luminous radiation terminates is taken to be $V_{\mathrm{e}}=6 \mathrm{~km} / \mathrm{sec}$, and the parameter of ablation to be $\sigma=2 \times 10^{-12} \mathrm{sec}^{2} / \mathrm{cm}^{2}$. This low value of the parameter of ablation is chosen in order to compensate for the influence of thermal energy loss by the particles at great heights, and the influence of shielding of the particles by meteor vapor and by air at lower heights.*

For the 'standard' particles with heights of separation of the order of $100 \mathrm{~km}$ the length of path reaches some kilometers. But it is only some meters for those with heights of separation of the order of $40-50 \mathrm{~km}$ (Figure $1^{* *}$ ). For the particles with given $r_{0}$ and $H_{1}$, the lifetime $T$ steadily increases with decreasing initial velocity, whereas the path length shows a maximum within small initial velocities (at $V_{0} \approx 15 \mathrm{~km} / \mathrm{sec}$, if $V_{\mathrm{e}}=6 \mathrm{~km} / \mathrm{sec}$ ).

The luminosity curves of particles, $I(H)$, correspond to the final part of the 'standard' luminosity curve of a meteor that is generated by a body passing the point of separation with a size and velocity equal to that of the particle at the moment of

\footnotetext{
* In the absence of this influence, the parameter of ablation of very hot particles is $\sigma=8 \times 10^{-12}$ $\sec ^{2} / \mathrm{cm}^{2}$.

** Figure 1 and the following are illustrations not only for the 'standard' particles, but for particles with any $r_{0}, \delta, \cos z$. If $r_{0}$, or $\delta$, or $\cos z$ is $n$ times that of the 'standard' particles, the scale $H_{1}$ must be shifted for $-15 \lg n$, in kilometers.
} 


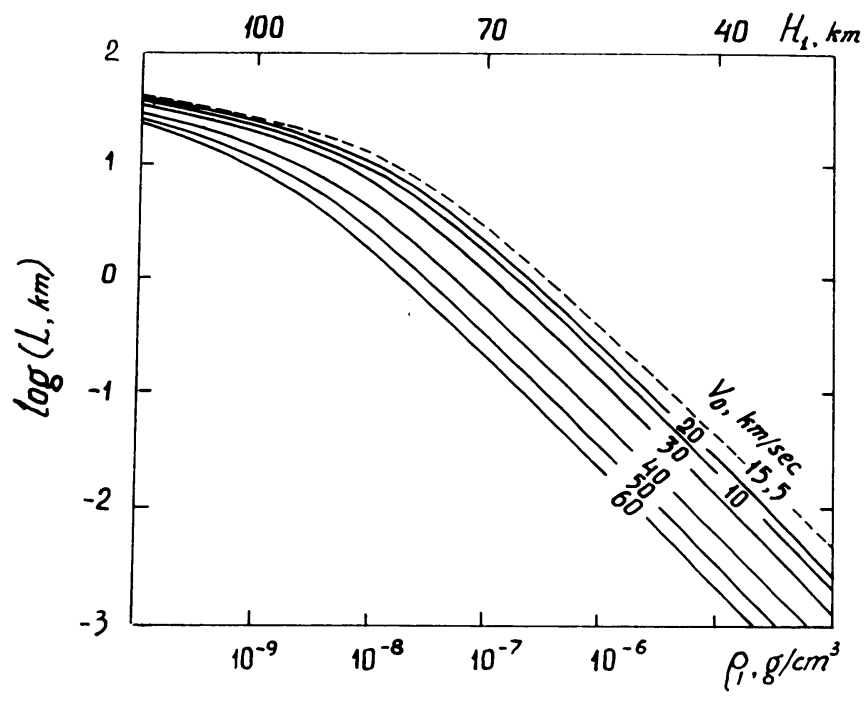

Fig. 1.

separation. Hence, the luminosity curve, even of a very small particle with great height $H_{1}$, is similar to the 'standard' one. At low heights the initial part of the 'standard' curve is eliminated and there is a shift of the beginning point to brighter magnitudes (Figure 2).

The deceleration of the particles depends strongly on the height of separation: it is small at great heights and reaches some tens or hundreds of $\mathrm{km} / \mathrm{sec}^{2}$ at heights in the range $60-70 \mathrm{~km}$ (Table 1). The separated particles quickly fall behind the meteor body, and the main part of their lifetime is beyond the influence of shielding by the meteor vapors of the main mass (Table 2).

The greater the height of separation the more will be the distance $\Delta l$ between the particle and the body to the end of its lifetime. The distance $\Delta l$ of small velocity particles increases more slowly, but reaches greater values (Figure 3). So the 'standard' particle with the height of separation of the order of $100 \mathrm{~km}$ and with the initial velocity $V_{0}=30 \mathrm{~km} / \mathrm{sec}$ reaches a distance from the body at the end of its lifetime of about $1 \mathrm{~km}$, but with $V_{0}=50 \mathrm{~km} / \mathrm{sec}$ the distance is less than $0.2 \mathrm{~km}$. Hence, if meteor wakes are originated by uniform particles, longer wakes must be observed for meteors of low velocities, assuming the meteors have the same magnitude. This is in fact observed (Jacchia et al., 1967).

The curves of particle luminosity $I$ vs. time $t$ after separation are contrasted to the curves $I$ vs. distance $\Delta l$ of the particle from the meteor body (Figure 4 ). The curves $I(\Delta l)$ drop more rapidly than curves $I(t)$. This is because the particles have a velocity close to the initial one during most of their lifetime. The difference is more distinct for particles with small initial velocities. This may be responsible for the discrepancy 


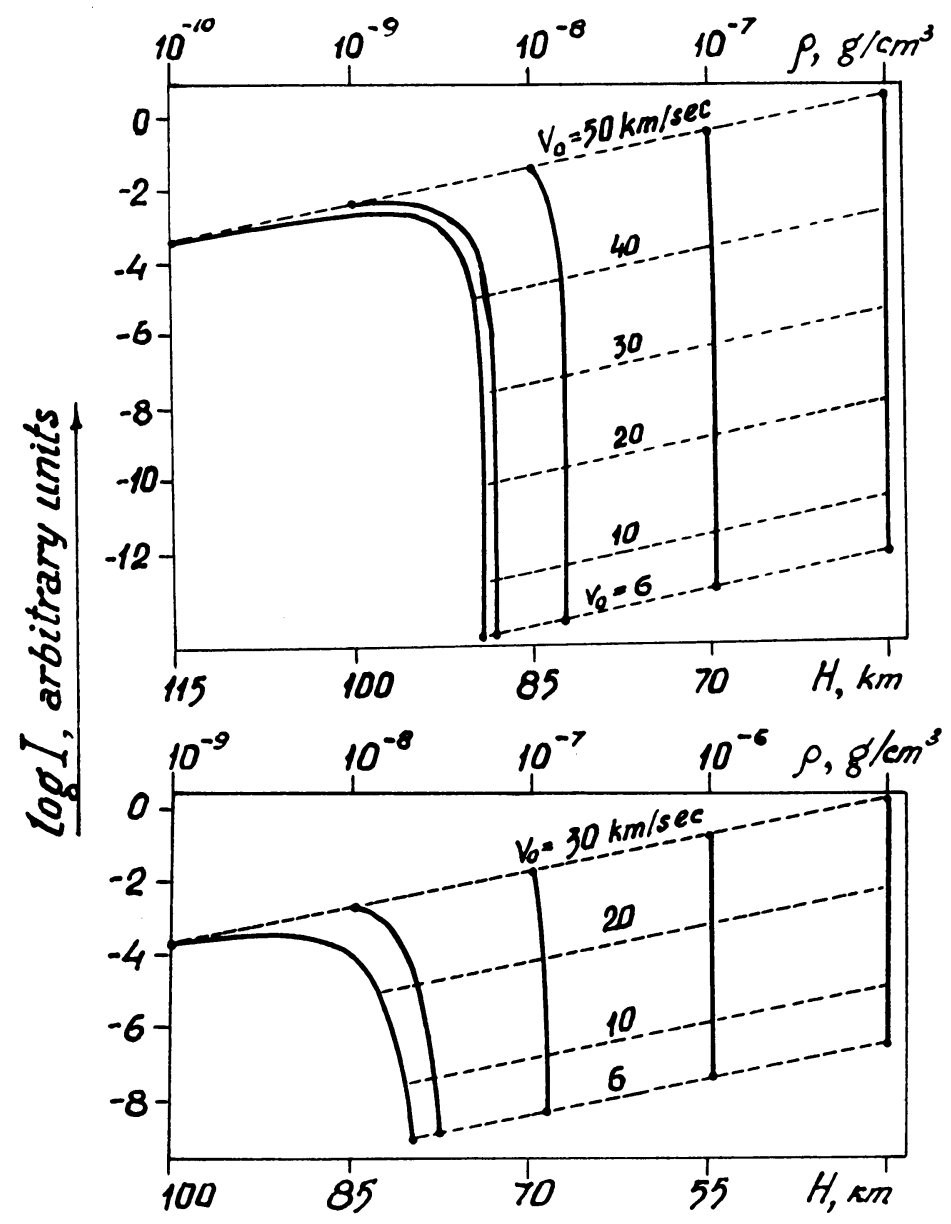

FIG. 2.

\section{Table 1}

The deceleration of a 'standard' particle just after separation $\left(\mathrm{km} / \mathrm{sec}^{2}\right)$

$\begin{array}{rrrrrrr}V_{0}(\mathrm{~km} / \mathrm{sec}) & 60 & 50 & 40 & 30 & 20 & 10 \\ H_{1}(\mathrm{~km}) & & & & & & \\ 100 & 7 \cdot 7 & 5 \cdot 4 & 3 \cdot 4 & 1 \cdot 9 & 0 \cdot 9 & 0 \cdot 2 \\ 85 & 77 \cdot 1 & 53 \cdot 6 & 34 \cdot 3 & 19 \cdot 3 & 8 \cdot 6 & 2 \cdot 1 \\ 70 & 771 & 536 & 343 & 193 & 85 \cdot 8 & 21 \cdot 4 \\ 55 & 7710 & 5360 & 3430 & 1930 & 858 & 214\end{array}$


Table 2

The distance $\Delta l$ of a 'standard' particle from the meteor body

$$
\left(H_{1}=85 \mathrm{~km}\right)
$$

$V_{0}(\mathrm{~km} / \mathrm{sec})$

60
50
40
30

\begin{tabular}{cl}
\multicolumn{2}{c}{$t=5 \times 10^{-4} \mathrm{sec}$} \\
$\Delta l(\mathrm{~mm})$ & $M / M_{0}$ \\
& \\
9.4 & 0.95 \\
6.7 & 0.95 \\
4.3 & 0.985 \\
2.4 & 0.995
\end{tabular}




\section{$\underline{V_{0}}=60 \mathrm{~km} / \mathrm{sec}$}

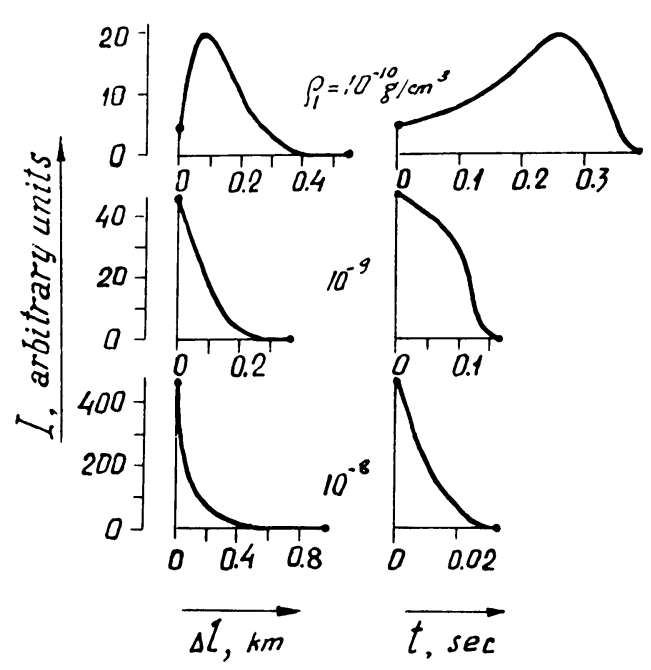

$\underline{V_{0}}=30 \mathrm{~km} / \mathrm{sec}$

FIG. 4.

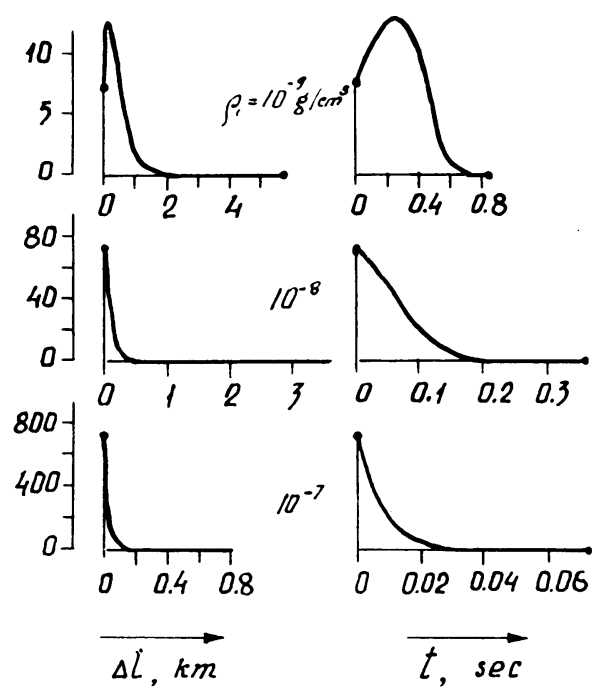

The long trajectories of particles separated high in the atmosphere affect the luminosity curves of meteors, and therefore influence the apparent density of meteor bodies and the parameter of ablation. In the case of the steady separation of particles, deceleration of a meteor as determined on the basis of analysis of photographic data just equals the deceleration of the meteor body, and the photometric mass $m_{\mathrm{ph}}$ is more than the mass of meteor body $m_{\mathrm{b}}$ because it is determined on the basis of light originated by molecules evaporated both from the meteor-body surface and from particle surfaces. If the particles are very small, the discrepancy between $m_{\mathrm{ph}}$ and $m_{\mathrm{b}}$ can be significant, even in the case of a small integrated mass of all particles. $\delta_{\text {obs }}$ is less than the actual density of the meteor body. If deceleration of the meteor during the lifetime of the particles is changed by a negligible amount, $\delta_{\text {obs }}$ is close to the density of the meteor body. In the course of an analysis of meteor photographs, the order of the discrepancy between $\delta_{\text {obs }}$ and density of the meteor body must be determined individually for each meteor. In our opinion, we can start from the assumption that the particles are uniform and that their initial size is of the order of $150 \mu$ (see below).

The parameter of ablation is given by the formula

$$
\sigma=2 \frac{\ln m_{a}-\ln m_{b}}{V_{a}^{2}-V_{b}^{2}}
$$




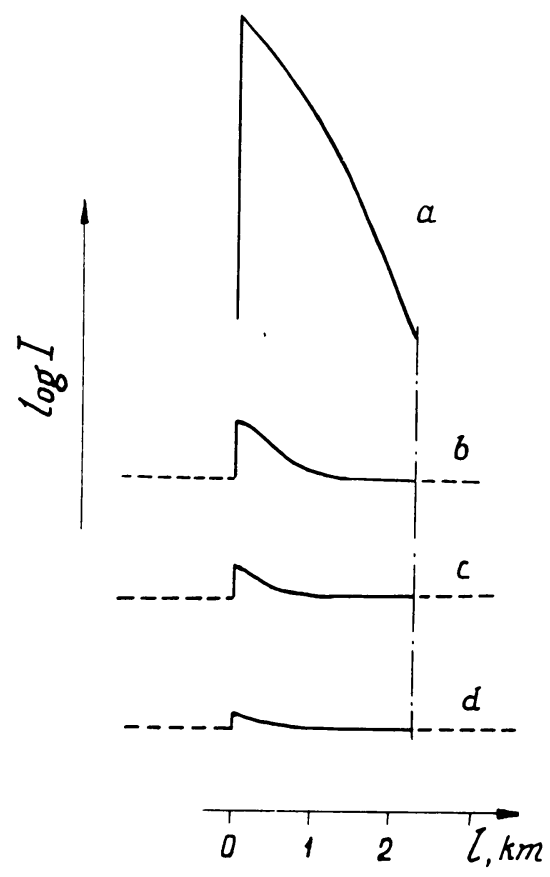

FIG. 5. (a) Luminosity curve of a 'standard' particle $\left(H_{1}=70 \mathrm{~km}, V_{0}=20 \mathrm{~km} / \mathrm{sec}\right)$; (b-d) luminosity curves of flares originated by different numbers of particles of this kind.

where the subscripts $a$ and $b$ specify the values of the mass and velocity at two points on the path. The observed velocity just equals the meteor body velocity. The discrepancy between $m_{\mathrm{ph}}$ and $m_{\mathrm{b}}$ is as above. The value of $\sigma_{\mathrm{obs}}$ is close to the parameter of ablation of the main body, when $m_{\mathrm{ph}} \approx m_{\mathrm{b}}$, or when the distance between points $a$ and $b$ is far greater than the length of the path of the particles. This can be expected at the end of meteor trajectories. On other parts of trajectories $\sigma_{\text {obs }}$ can be either greater than or smaller than the parameter of ablation of the main body.

The index of fragmentation (Jacchia et al., 1967) is defined by

where

$$
\chi=\frac{\mathrm{d}}{\mathrm{d} s} \lg \frac{\left(\begin{array}{l}
\mathrm{d} v \\
\mathrm{~d} t
\end{array}\right)_{\mathrm{obs}}}{\left(\frac{\mathrm{d} v}{\mathrm{~d} t}\right)_{\mathrm{th}}}
$$

$$
s=\lg \frac{m_{0}-m}{m}
$$

This index is said to be non-zero only in the case of progressive fragmentation 
(Jacchia, 1955; Jacchia et al., 1967). Indeed, in the case of separation of small particles, $\chi$ would be equal to zero if the particles would be evaporated instantaneously. But this is not the case. The consequence is that the meteor body mass drops more rapidly than the photometric mass, and this leads to $\chi \neq 0$.

The instantaneous spray of a large number of small particles is of interest. At the beginning of the trajectories of meteors, it gives rise to humps on the luminosity curves, at the end of trajectories, to flares. The flares make possible the determination of the size of the particles. Attempts of such a determination have been made by Smith (1954) and by Ivanikov (1965), but their methods have deficiencies, e.g. the discrepancy between the observed length of the flares and the length of the path of the particles covered during their lifetime was not taken into account (Figure 5). The

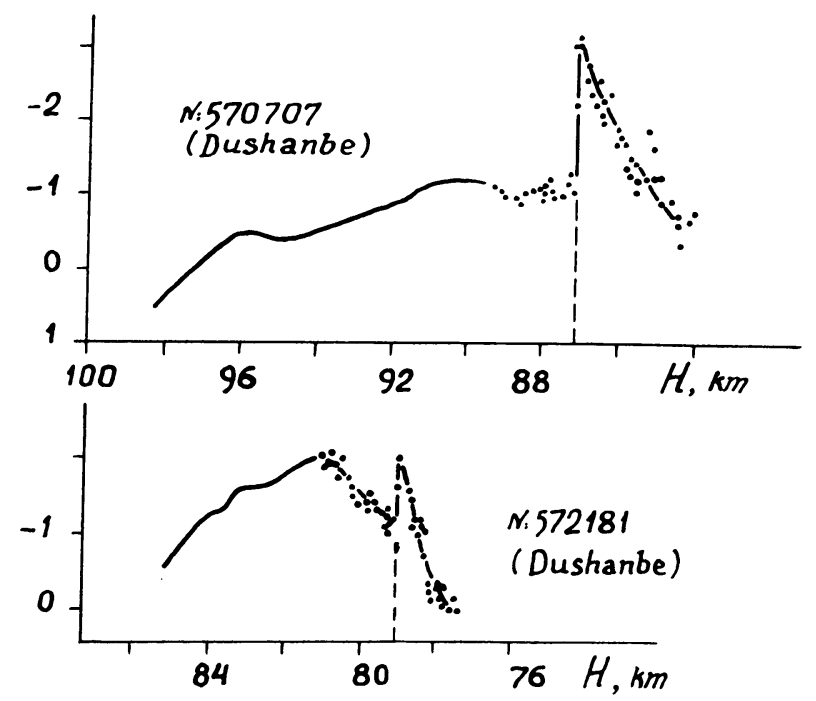

Fig. 6. The luminosity curves $M$ vs. $H$ of two meteors with flares.

discrepancy is a consequence of a very rapid drop of luminosity of the particles at the end of their lifetime and can be considerable, especially for particles with small and average velocities at great heights.

On the basis of the analysis of 108 flares on meteor photographs (mainly from the collection of the Astrophysical Institute, Dušanbe) we determined the size of separated particles. The selection of flares with an abrupt growth of luminosity at the beginning of the flare (close to vertical on the luminosity curves) and a smooth decrease without noticeable irregularities, permits a decrease in the number of parameter values which have to be assumed. The method of reduction is described elsewhere (Simonenko, 1967).

In the course of computations, the particles are supposed to be uniform, of spherical form, and with $\delta=3.5 \mathrm{~g} / \mathrm{cm}^{3}$. Bearing in mind the shielding of the particles, the para- 
meter of ablation is chosen equal to $2 \times 10^{-12} \mathrm{sec}^{2} / \mathrm{cm}^{2}$.* Hence, the determined size of particles, i.e. the initial radius $r_{0}$, is the effective one.

The result is that the mean effective radius of separated particles is about $70 \mu$. The dispersion of $r_{0}$ is small: $80 \%$ of all flares give $30 \mu<r_{0}<110 \mu$. In the case of a normal distribution of $r_{0}$, this corresponds to a standard deviation in $r_{0}$ of about $33 \mu$. Bearing in mind the errors of such parameters as velocity, height of separation, etc., the standard deviation of the initial size of particles may decrease to $20 \mu$, for a normal distribution of sizes. Thus during all flares'particles of almost uniform size are separated.

The small dispersion of $r_{0}$ is responsible for the dependence of the heights of flares on the velocities of meteors (Ivanikov, 1965; Jacchia, 1949), for decreasing of the length of flares near the end of meteor trajectories (Ivanikov, 1965; Jacchia, 1949), and for the incorrect concept that an instantaneous spray of particles takes place only at the end of meteor trajectories.

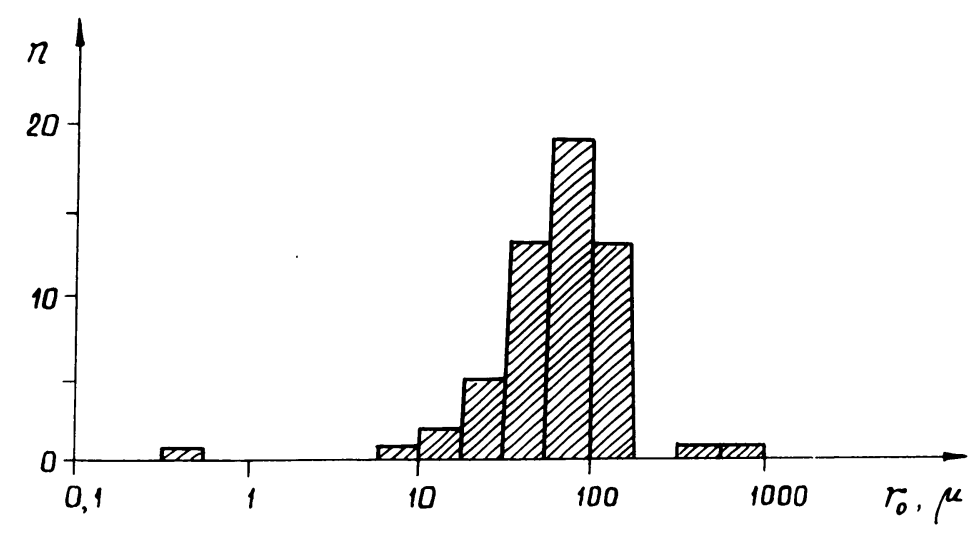

FIG. 7.

On the basis of the examination of meteors with a high tendency to flare, we conclude that the distance between flares, as well as the length of flares, decreases toward the end of meteor trajectories. The mass of air intercepted per unit of meteor body surface during intervals between the flares is roughly constant. We evaluated the depth of the layer of the meteor body evaporated during these intervals and concluded that it is of the order of some tens of microns in the case $\delta=3.5 \mathrm{~g} / \mathrm{cm}^{3}$ and $\sigma=2 \times 10^{-12} \mathrm{sec}^{2} / \mathrm{cm}^{2}$.

Thus, we are inclined to conclude that particles of a size of the order of $150 \mu$ appear to be structural elements (or grains) of meteor bodies. In our opinion, their separation takes place not only during flares but during the entire time of meteor

* The thermal radiation at heights of flares is negligible. 
flight in the atmosphere, and is a typical process of fragmentation for meteor bodies. Thermal stresses will be significantly greater at grain boundaries, and will favor the separation of grains.

\section{References}

Ivanikov, V.I. (1965) Bjull. Inst. Astrofiz. Dušanbe, 39-40, 3.

Jacchia, L. G. (1949) Harv. Coll. Obs. Techn. Rep., No. 3.

Jacchia, L. G. (1955) Astrophys. J., 121, 121.

Jacchia, L.G., Verniani, F., Briggs, R.E. (1967) Smithson. Contr. Astrophys., 10, 1.

Kramer, E.N. (1965) Geomagnetizm i aeronomija, 5, 276.

Levin, B.Ju. (1961) Bjull. Kom. Komet. Meteor., 6, 3.

McCrosky, R.E. (1958) Astr. J., 63, 97.

Simonenko, A.N. (1967) Komety $i$ Meteory, 15, 34.

Simonenko, A.N. (1968) Astr. Zu., 45 (in press).

Smith, H.J. (1954) Astrophys. J., 119, 438.

Stepanov, D.I. (1965) Paper presented at the XIth Meeting of the Comm. for Comets and Meteors of the Astr. Council of the U.S.S.R., Sept. 1965.

Whipple, F.L. (1950) Proc. nat. Acad. Sci. Am., 36, 687.

Whipple, F. L. (1951) Proc. nat. Acad. Sci. Am., 37, 19.

\section{DISCUSSION}

Fedynskij: This is the first very interesting attempt to evaluate the size of the elementary particles of which meteoroids consist. 